\title{
Interpretation through experimental simulations of phase functions revealed by Rosetta in 67P/Churyumov-Gerasimenko dust coma
}

\author{
A. C. Levasseur-Regourd ${ }^{1}$, J.-B. Renard ${ }^{2}$, E. Hadamcik ${ }^{3}$, J. Lasue ${ }^{4}$, I. Bertini ${ }^{5}$, and M. Fulle \\ ${ }^{1}$ LATMOS, Sorbonne Université, CNRS, UVSQ, Campus Pierre et Marie Curie, 4 place Jussieu, 75005 Paris, France \\ e-mail: aclr@latmos.ipsl.fr \\ ${ }^{2}$ LPC2E-CNRS, 3A avenue de la Recherche Scientifique, 45071 Orléans cedex 2, France \\ ${ }^{3}$ LATMOS/IPSL, UVSQ Université Paris-Saclay, Sorbonne Université, CNRS, Guyancourt, France \\ ${ }^{4}$ IRAP, Université de Toulouse, CNRS, CNES, UPS, 9 avenue Colonel Roche, 31400 Toulouse, France \\ 5 Department of Physics and Astronomy G. Galilei, University of Padova, Vic. Osservatorio 3, 35122 Padova, Italy \\ ${ }^{6}$ INAF - Osservatorio Astronomico, Via Tiepolo 11, 34143 Trieste, Italy
}

Received 16 December 2018 / Accepted 5 March 2019

\begin{abstract}
Context. The dust-brightness phase curves that have been measured by the OSIRIS cameras on board the Rosetta spacecraft within the coma of comet 67P/Churyumov-Gerasimenko (67P) present a remarkable flattened u-shape.

Aims. Our goal is to compare these phase curves with those of tentatively analog dust samples to assess the key dust properties that might induce this shape.

Methods. Light-scattering measurements have been made with the PROGRA2 instrument in the laboratory and in microgravity conditions on samples of different physical properties and compositions that are likely to be representative of cometary dust particles.

Results. We find that the brightness phase curves of a series of interplanetary dust analogs that have been recently developed (to fit the polarimetric properties of the inner zodiacal cloud and their changes with heliocentric distance) are quite comparable to those of 67P. Key dust properties seem to be related to the composition and the porosity.

Conclusions. We conclude that the shape of the brightness phase curves of $67 \mathrm{P}$ has to be related to the presence of a significant amount of organic compounds (at least 50\% in mass) and of fluffy aggregates (of a size range of 10-200 $\mu \mathrm{m}$ ). We also confirm similarities between the dust particles of this Jupiter-family comet and the particles within the inner zodiacal cloud.
\end{abstract}

Key words. comets: general - comets: individual: 67P/Churyumov-Gerasimenko - scattering - polarization instrumentation: miscellaneous - space vehicles: instruments

\section{Introduction}

Everywhere in the solar system, dust media, such as cometary comae or the interplanetary dust cloud, scatter solar light. While the properties of the scattered light depend on the properties of the scattering medium, it is a complex inverse problem to derive information from light-scattering measurements. The unique Rosetta rendezvous with comet 67P/Churyumov-Gerasimenko (thereafter 67P) has fortunately provided a wealth of information on cometary dust particles during its 26-month-long mission, including phase functions of these particles (Bertini et al. 2017).

From past cometary flybys, including those of comet $1 \mathrm{P} /$ Halley in 1986 , to the $67 \mathrm{P}$ rendezvous, numerous observations of dust in cometary comae have been obtained from the Earth and Earth-orbiting observatories. On a given day, remote observations of a comet correspond to a given phase angle $\alpha$ and to different distances to the nucleus within a possibly heterogeneous coma. Retrieving a phase curve thus requires extended observational periods, corresponding to significant changes in solar distance and possible changes in activity during outburst events (Dollfus et al. 1988). Interpreting these phase curves nevertheless provides information on the dust properties of various comets, with realistic experimental and numerical simulations now validated through 67P ground-truth.

The Rosetta mission has provided a huge amount of data on dust particles and their phase curves over a wide range of distances to the Sun and to the nucleus. Results stem from innovative dust instruments, the COmetary Secondary Ion Mass Analyzer (COSIMA), the Grain Impact Analyzer and Dust Accumulator (GIADA), the Micro-Imaging Dust Analyzis System (MIDAS), as well as from its Optical, Spectroscopic and Infrared Remote Imaging System (OSIRIS), and its Philae lander (Glassmeier et al. 2007). Rosetta dust studies, obtained on a wide range of sizes (at least from tens of nanometers to a few meters) have clearly established what had been suggested after Giotto and Stardust missions (Fulle et al. 2000; Hörz et al. 2006; Matrajt et al. 2008): dust particles are aggregates that present a hierarchical structure and have an average bulk porosity of at least $60 \%$ (Hilchenbach et al. 2016; Bentley et al. 2016; Langevin et al. 2016; Mannel et al. 2016). The Rosetta mission has also provided outstanding information on the composition of refractories in the coma, and it has shown that complex organic molecules represent a significant component of the solid fraction of comets (Goesmann et al. 2015; 
Fray et al. 2016, 2017; Bardyn et al. 2017). These results (see, e.g., Levasseur-Regourd et al. 2018, for a review) are of major interest for the interpretation of remote observations of dust in the comae of comets, and especially of Jupiter-family comets (JFCs) such as 67P. It may be added that while cometary dust and dust from asteroids replenish the interplanetary dust cloud (also called zodiacal cloud), JFCs have been understood based on various observational and dynamical approaches to be the main sources of dust particles along the Earth's orbit (Lasue et al. 2007; Nesvornỳ et al. 2010; Rowan-Robinson \& May 2013; Carrillo-Sánchez et al. 2016).

In relation with optical properties of dust, OSIRIS multiwavelength observations (from about 376 to $744 \mathrm{~nm}$ ) have allowed Bertini et al. (2017) to retrieve the shape of the coma-dust phase function between about $15^{\circ}$ and $150^{\circ}$ from data obtained between March 2015 and February 2016. The reflectance slowly decreases from about $20^{\circ}$ to $100^{\circ}$ before increasing again without any sharp surge in the forwardscattering region. This flattened $\mathrm{u}$-shape seems to agree with most observations of other comets (Bertini et al. 2017, Fig. 5) and rules out a strong forward-scattering.

OSIRIS phase curves of the coma dust have a significantly flatter decrease in backscattering than the nucleus phase curves (Fulle et al. 2018). They provide clues to a slight reddening for dust particles, lower than for the nucleus, and to a negligible phase reddening, which is consistent with the absence of multiple scattering in the coma (Bertini et al. 2017; Fulle et al. 2018). It may finally be added that a phase curve (from $1.2^{\circ}$ to $74^{\circ}$ ) has also been derived from OSIRIS data obtained between July 2014 and February 2015, typically at $612.5 \mathrm{~nm}$ (Fink \& Doose 2018). It shows a fair agreement with the results described above in the $15^{\circ}-90^{\circ}$ domain of overlapping phase angles.

Some characteristics of the light scattered by the randomly polarized solar light on dust media (such as cometary comae or the interplanetary dust cloud) may provide information on their properties. Characteristics of scattered light typically depend upon the concentration, size, size distribution, shape, morphology, porosity, and complex refractive index (related to the composition) of the dust particles, and thus upon their geometric albedo. Because these particles are mostly larger than the observational wavelengths (usually in the visible and near-infrared domains), the Mie theory cannot be used, except for almost spherical dust particles or droplets.

Simulations are mandatory to try to unequivocally interpret observational data in terms of the physical properties and possibly the composition of the dust particles. Observations of the linear polarization of the scattered light are somewhat easier to interpret than brightness observations because they neither depend upon the distances of the scattering medium to the Sun and to the observer nor upon the concentration of the medium. For cometary comae of comets that have been extensively observed (1P/Halley and C/1995 O1 Hale-Bopp), numerical and experimental simulations have typically suggested that the dust particles are likely to be fluffy aggregates of irregular grains mixed with some compact particles, and that they are composed of minerals and absorbing carbonaceous material (Hadamcik et al. 2007; Levasseur-Regourd et al. 2008; Lasue et al. 2009). Similar approaches have also been developed for the interplanetary dust cloud (Lasue et al. 2007; Hadamcik et al. 2019).

In this paper, we first consider experimental simulations with analogs that might be representative of cometary dust. The simulations have been mostly performed under microgravity conditions with the PROGRA2 instruments. We then compare the phase curves of the analogs with those of dust particles in the coma of 67P just before and soon after perihelion to point out satisfactory analogs. We then discuss our results and their possible implications.

\section{PROGRA2 experimental simulations of light scattering with cometary analogs}

Experimental simulations in the laboratory or under microgravity conditions allow measurements to be made on various samples of controlled characteristics. Reviews on technical developments for light-scattering measurements in microgravity conditions, together with types of samples of interest in planetary sciences, and on experimental scattering matrices of clouds of randomly oriented particles may be found in Levasseur-Regourd et al. (2015) and in Muñoz \& Hovenier (2015), respectively. We summarize below the instrumentation we have used and the samples we have estimated to be of interest for simulating cometary dust properties.

\subsection{PROGRA2 instrumentation}

The PROGRA2-Vis instrument (PRopriétés Optiques des GRains Astronomiques et Atmosphériques-Visible, i.e., Optical properties of astronomical and atmospheric grains in the visible domain) is dedicated to the retrieval of the brightness and linear polarization phase functions of levitating particles with random orientations (Worms et al. 1999; Renard et al. 2002). The light sources at present are halogen white lamps with a depolarizing filter and a spectral filter; one source operates at $555 \pm 30 \mathrm{~nm}$, and the other at $650 \pm 30 \mathrm{~nm}$. An optical fiber carries the light to the vial in which the particles are lifted. The particles that cross the light-beam scatter the incident light. A polarizing beam-splitter cube splits the scattered light into its two components, parallel and perpendicular to the scattering plane. These are recorded by two synchronized cameras with similar fields of view. The vial and a third synchronized camera are mounted on a rotating device; the incident light beam and the vial rotate to change the phase angle in the $8^{\circ}-165^{\circ}$ range, the detection system being in a fixed position. The third camera records the scattered light at a constant phase angle of $90^{\circ}$, and acts as a reference camera. The polarization is retrieved from the two first cameras; the brightness is retrieved after normalization of the flux recorded by the two first cameras to the flux of the third camera. In the following, we consider the brightness results for further comparisons.

The levitation of the particles is obtained by two methods. For compact particles smaller than about $20 \mu \mathrm{m}$ and for fluffy particles, which are aggregates of submicron-sized grains (also called monomers), the particles are lifted through an air draught technique (Hadamcik et al. 2002). For compact particles larger than $20 \mu \mathrm{m}$ and for mixtures of particles with different structures, the measurements are conducted in microgravity conditions during parabolic flights on board the A300 ZeroG or the A310 ZeroG aircraft managed by the Novespace Company (Renard et al. 2002).

\subsection{Choice of samples}

Four types of samples that could partly reproduce some physical properties of cometary material have been chosen. Scanning electron microscope (SEM) images of these samples are shown on Fig. 1.

Samples with morphologies that might be representative of cometary dust are considered first. Black carbon fluffy 

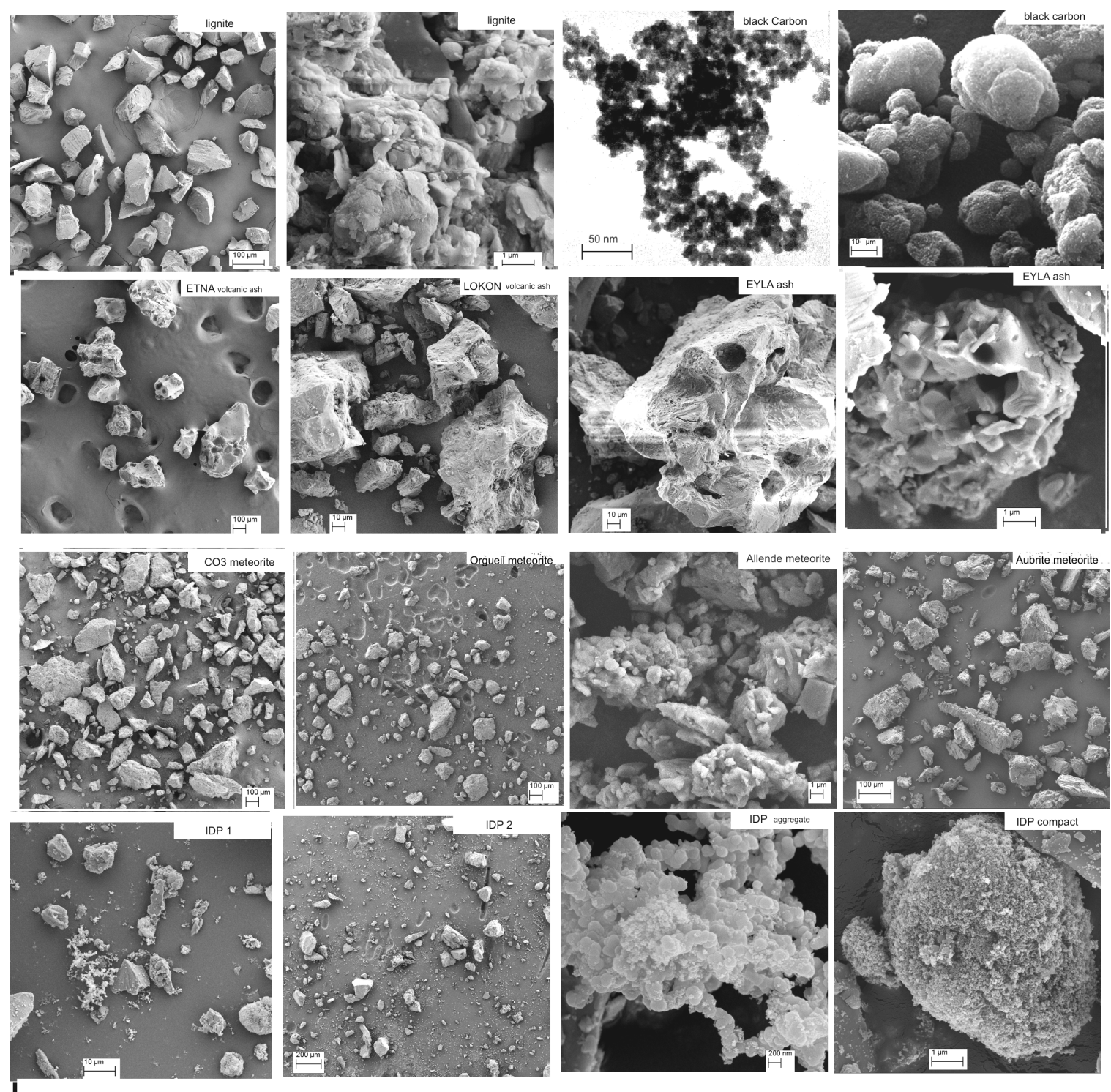

Fig. 1. Typical SEM images of dust samples that might be representative of cometary dust properties in terms of size, morphology, and composition. From top to bottom, we present the black carbon fluffy aggregates, the porous volcanic ashes, the crushed meteoritic dust particles, and the interplanetary dust analogs that we use for comparisons with OSIRIS phase functions.

aggregates, with a mean size of about $100 \mu \mathrm{m}$, of monomers of $14 \mathrm{~nm}, 25,56$ and $90 \mathrm{~nm}$ may be used to estimate the effect of the monomer sizes on the shape of the phase function (Francis et al. 2011, updated from). Second, porous volcanic ashes from Eyjafjallajökull (Iceland) and Lokon (Indonesia) with sizes below $50 \mu \mathrm{m}$ and also from Etna (Italy), with three size-ranges of about 50, 100 and $200 \mu \mathrm{m}$, are considered as possibly representative of porous particles.

Then, samples with compositions tentatively representative of cometary dust are considered. Four samples of crushed meteoritic dust particles were obtained, with sizes smaller than $200 \mu \mathrm{m}$, one from an aubrite (i.e., achondrite meteorite) from Antarctica, and, possibly more representative of the composition, carbonaceous chondrites, such as Orgueil (CI1), Allende (CV3), or North Africa 6352 (CO3) (Hadamcik et al. 2011); the size of the crushed particles is smaller than $200 \mu \mathrm{m}$. Finally, four possible interplanetary dust analogs, composed of mixtures of carbonaceous and mineral compounds, with ratios that change from one analog to the other, were used. While each material exists in different structures, the ratio between fluffy aggregates and more compact particles was kept constant in mass, $(35 \pm 10) \%$ for aggregates and $(65 \pm 10) \%$ for compact particles. While mass, volume, and count percentages certainly depend on the various porosities of the particles, values of about $37 \%$ in volume were derived for extremely porous fractal particles in 67P coma by Fulle \& Blum (2017). Moreover, about $35 \%$ in counts of type $\mathrm{C}$ tracks of dust particles collected by Stardust within the coma of 81P/Wild 2 were found by Burchell et al. (2008). Carbonaceous particles are fluffy carbon-black aggregates with submicron-sized grains or porous coals such as lignite; mineral particles are silicates in fluffy aggregates or in more compact particles, and some of them originate from crushed meteorites (Hadamcik et al. 2019). The particles follow size distributions of $10-320 \mu \mathrm{m}$. The percentage of carbonaceous content decreases from 60 to $30 \%$ to tentatively reproduce the evolution with solar distance of the optical properties of the cloud and thus of the particles composition (Lasue et al. 2007). 


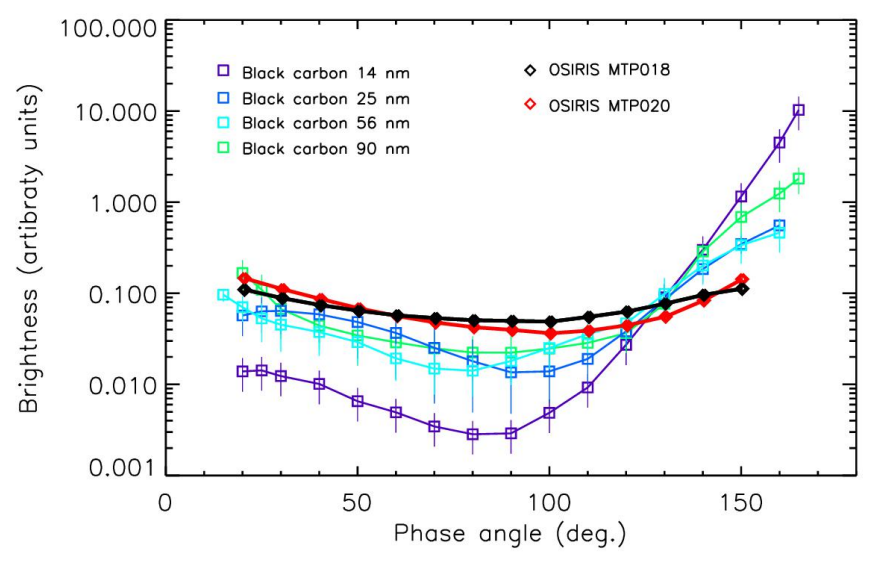

Fig. 2. Comparison between OSIRIS and PROGRA2 phase functions for aggregates of black carbon particles with different sizes.

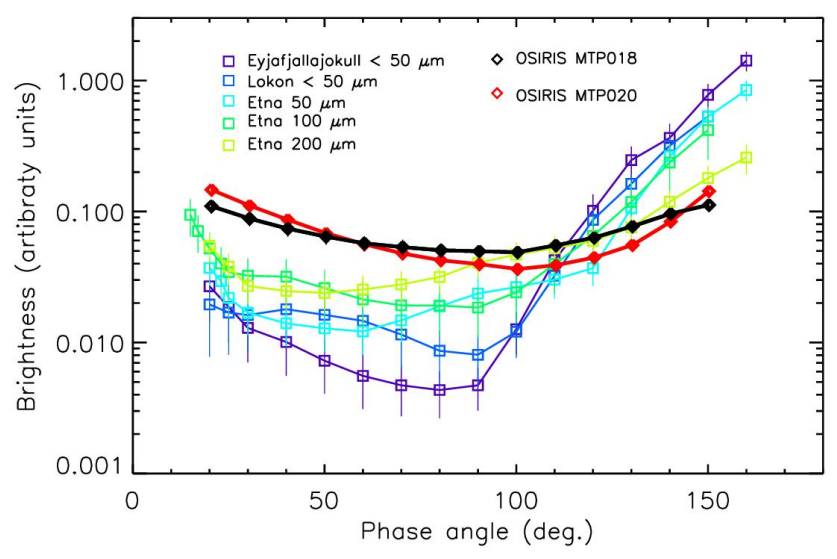

Fig. 3. Comparison between OSIRIS and PROGRA2 phase functions for porous volcanic particles of different sizes.

\section{Comparison between dust-coma phase functions of 67P and those obtained for analogs}

The brightness phase curves measured for the samples described above do not present significant changes with wavelength in the visible domain. Together with their errors bars, they are presented in Figs. 2-5 for black carbon fluffy aggregates, porous volcanic ashes, crushed meteoritic dust particles, and finally for interplanetary dust analogs, respectively. The OSIRIS phase curves (Bertini et al. 2017), typically obtained in the green domain (by $537 \mathrm{~nm}$ ) with the wide-angle camera up to $150^{\circ}$ phase angle, and in the orange domain (by $649 \mathrm{~nm}$ ) with the narrow-angle camera up to $140^{\circ}$ phase angle, are also quite comparable. Because the green measurements cover a wider angular coverage, we have chosen to compare them with our laboratory data in the green domain. We have considered two sets of data with remarkably small errors bars, MTP018 on 7 July 2015 and MTP020 on 28 August 2015, at heliocentric distances of 1.32 au and $1.25 \mathrm{au}$, respectively, and at distances to the nucleus of 153 and $420 \mathrm{~km}$, respectively.

It is necessary to normalize the OSIRIS brightness phase functions and those from PROGRA2 for comparison purposes. The OSIRIS data have been divided by the sum of the intensities. The PROGRA2 data have also been divided by the sum of the intensity in the $20^{\circ}-150^{\circ}$ range to cover the angular range of OSIRIS. Figures 2-5 allow immediate comparisons between

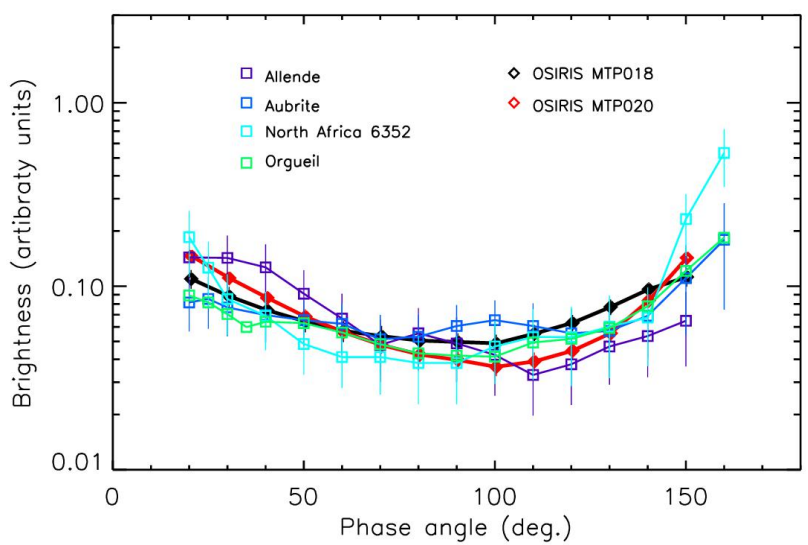

Fig. 4. Comparison between OSIRIS and PROGRA2 phase functions for various dust particles from crushed meteorites.

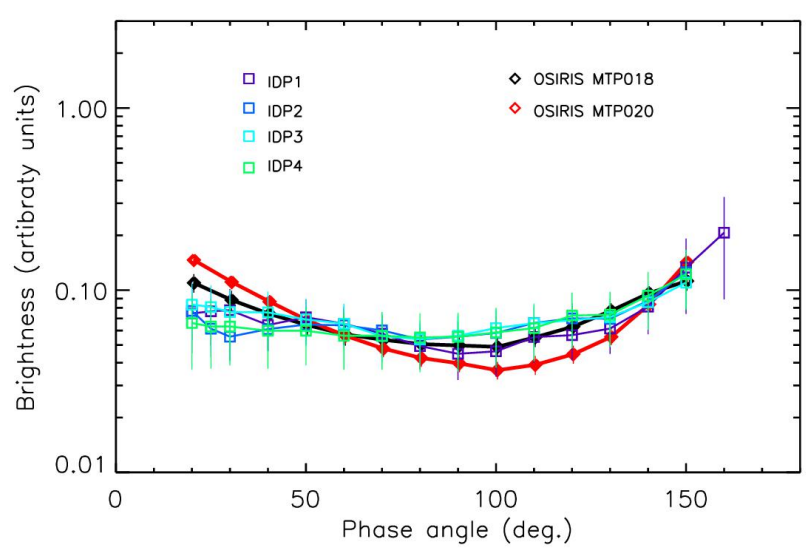

Fig. 5. Comparison between OSIRIS and PROGRA2 phase functions for various interplanetary dust analogs.

PROGRA2 and OSIRIS measurements just before and soon after perihelion. Following the types of families described above, they are ordered from poor to better agreement with OSIRIS data.

The PROGRA2 measurements with black carbon aggregates do not reproduce the OSIRIS phase curves, although the discrepancies decrease with increasing grain sizes. The agreement is also poor for porous volcanic ashes for both small and large phase angles and for the range of phase angles at minimum brightness.

For crushed meteorites, all phase curves present the expected u-shape, with better fits for the crushed carbonaceous chondrites, and especially Orgueil, than for the crushed aubrite. Orgueil dust is brownish and is made of porous particles and opaque agglomerates. CO3 6352 is also brownish and is made of irregular relatively porous fragments. Allende fragments are grayer, irregular, and more compact. Aubrite is clear gray with rather compact fragments.

Satisfactory agreements are obtained for the interplanetary dust analogs, although their phase curves are relatively flat in the backscattering region. They provide the expected flattened u-shape phase curves.

More precisely, standard deviations are high on average for black carbon $(\approx 0.158)$ and for volcanic ashes $(\approx 0.125)$. They are significantly lower for crushed meteorites $(0.025$, with only 0.014 for Orgueil) and for interplanetary dust analogs (0.021). 


\section{Discussion}

\subsection{Significance of the different fits}

The comparison between the brightness phase curves obtained by OSIRIS (before and after perihelion in July-August 2015) and the phase curves obtained by the PROGRA2 instrument on dust samples that might be representative of cometary dust indicates that black carbon fluffy aggregates, porous volcanic ashes, or some crushed meteoritic samples, such as the aubrite and Allende or North Africa 6352 samples do not provide satisfactory fits. Allende, as also noted in Muñoz et al. (2000), presents significant backscattering and a minimum in brightness above $90^{\circ}$ phase angle.

However, a satisfactory fit is found for a crushed Orgueil sample, which is a sample from a carbonaceous meteorite, with a porous structure built of aggregates in a size range of tens of micrometers (Tomeoka \& Buseck 1988). A satisfactory fit is also found for samples that have been proposed as analogs for interplanetary dust particles. To our knowledge, they correspond to the first laboratory measurements that show a clear u-shaped phase curve, using tentative cometary dust analogs.

The somewhat satisfactory fits are better before than after perihelion; the typical standard deviation is 0.008 instead of 0.002 for the Orgueil sample and 0.013 instead 0.022 for the four interplanetary dust analogs. This might correspond to the fact that the population of dust particles that were released after perihelion was less homogeneous than before.

\subsection{Comparison with clues from the linear polarization of dust analogs}

Clues from brightness phase curves are, as described in the Introduction, more difficult to interpret than those of linear polarization phase curves, at least when multiple scattering is not significant. Linear polarization phase curves, $P(\alpha)$, usually present a small negative branch in the backscattering region and reach their maximum, $P_{\max }$, in the $80^{\circ}-100^{\circ}$ phase angle range. They have indeed been extensively used to try to estimate the properties of cometary and interplanetary dust particles, as reviewed in Kiselev et al. (2015) and Lasue et al. (2015), respectively. PROGRA2 polarization measurements can provide additional constraints for comparison.

Measurements on three meteoritic dust samples led to $P_{\max }$ values below $15 \%$, while the values obtained for the Orgueil sample were higher, about 35\% (Hadamcik et al. 2019). Previous measurements, made in 1998-1999, with similar Allende and Orgueil samples (Worms et al. 2000), have provided similar results. The $P_{\max }$ values derived from observations of cometary comae present some dispersion that might be related to cometary activity and to the observational field of view. $P_{\max }$ remains about $5-10 \%$ for the so-called low-polarization comets, and may reach up to $25-30 \%$ for the so-called high-polarization comets.

Measurements on the four interplanetary dust analogs led to $P_{\max }$ values in the $20-35 \%$ range. While polarimetric phase curves for the zodiacal cloud are quite difficult to obtain because observations along a line of sight correspond to changing phase angles and solar distances, inversion is possible in the near-ecliptic symmetry plane of the interplanetary dust cloud (Levasseur-Regourd et al. 2001). For a phase angle equal to $90^{\circ}$, $P_{\text {max }}$ progressively decreases with solar distance from about $35 \%$ at 1.5 au to about $28 \%$ at 1 au, and finally about $20 \%$ at 0.4 au. This trend, which has long been suspected to originate in partial sublimation of semi-volatile organics, is perfectly reproduced with the interplanetary dust analogs described above, with a decreasing percentage in organics (Hadamcik et al. 2019, Fig. 7). The agreement between the dust-brightness phase curves of $67 \mathrm{P}$ and those of our interplanetary dust analogs is logical, considering that JFCs are now recognized (see the Introduction) to be the main source of interplanetary dust along the Earth's orbit.

Finally, it may be added that although the trends of polarimetric phase curves point out changes in dust properties from the values of the near-maximum polarization (for phase angles in the $90^{\circ}-120^{\circ}$ range), brightness phase curves (when available) may be of major interest for comparisons with simulations results in the back- and forward-scattering regions.

\subsection{Comparison with numerical simulations}

Numerical simulations have been developed for the polarimetric phase curves of extensively observed comets, such as 1P/Halley and C/1995 O1 Hale-Bopp. Satisfactory fits to observations have been obtained over a wide range of wavelengths with aggregates of submicron-sized grains mixed with spheroidal particles. Both are consisting of absorbing organic-type material and feebly absorbing silicate-type material (Levasseur-Regourd et al. 2008; Lasue et al. 2009); interpretations of the linear polarization data for comets are reviewed in Kiselev et al. (2015).

In relation with brightness phase curves, it was noted in microgravity experiments that $\mathrm{u}$-shaped brightness phase curves for rather spherical glass beads (sizes about $50-150 \mu \mathrm{m}$ ) become flattened when the beads are covered with a thin layer of graphite. This shows that carbonaceous compounds may be important for the shape of the phase curve in intensity (Lasue et al. 2007). The rougher surface of the carbon-coated beads may also have an influence on this effect.

For observations of 67P, Moreno et al. (2018) have recently proposed based on numerical simulations that particles with sizes above about $20 \mu \mathrm{m}$, the complex refractive index of which is equal to $1.6+0.1 \mathrm{i}$ (dark absorbing particles at $0.6 \mu \mathrm{m}$ ) and the porosity of which is in the 60-70\% range, would fit the OSIRIS phase functions (Bertini et al. 2017), assuming that the particles are elongated (with long axes perpendicular to the solarradiation direction) and that they have various aspect ratios. Moreover, Markkanen et al. (2018) performed numerical simulations that also reproduced the OSIRIS data with irregular particles, with sizes ranging from 5 to $100 \mu \mathrm{m}$, that were composed of an intimate mixture of sub-micrometer organic material and micrometer-sized spherical silicate grains.

This means that Moreno et al. (2018) and Markkanen et al. (2018), although they investigated the free parameter space in their simulations in different ways, both indicate that the phase curves obtained from OSIRIS data in the inner coma (Bertini et al. 2017) can be representative of dust particles that are larger than a few microns or tens of microns, and that the absorbing component correspond to a very important part. This agrees with our laboratory results, where satisfactory fits with the observations are provided by irregular absorbing particles (e.g., tens of micrometer-sized crushed carbonaceous meteorites such as Orgueil and interplanetary dust analogs).

\section{Conclusions}

The dust-brightness phase curves obtained by the OSIRIS observations during the Rosetta rendezvous have revealed unique trends that can be compared with those measured on samples that might be representative of the properties of cometary dust particles. Light-scattering measurements obtained with the 
PROGRA2 instrument, either in the laboratory or in microgravity conditions, indicate that neither black carbon aggregates, built of grains in the range of 14-90 nm, nor porous volcanic ashes of various origins and sizes can fairly reproduce the flattened u-shape of the observational phase curves.

Excellent agreement is nevertheless obtained between the OSIRIS phase curves in July-August 2015 (and especially a few weeks before perihelion) and those measured either on a dust sample from Orgueil meteorite or on samples recognized to be satisfactory analogs for the bulk polarimetric scattering properties of interplanetary dust. The typical shape of the dust-brightness phase curves obtained within the coma of 67P/Churyumov-Gerasimenko is related to a significant amount of organic compounds (at least $50 \%$ in mass) and to fluffy aggregates with sizes ranging from 10 to $200 \mu \mathrm{m}$. These results may be slightly different after perihelion, possibly because dust particles of different sizes and structures were then released into the coma. The results nevertheless point out similarities between the dust particles of a Jupiter-family comet and those of dust particles within the inner zodiacal cloud.

Acknowledgements. A.C.L.R. and J.L. acknowledge support from Centre National d'Études Spatiales (CNES) in the scientific analysis of instruments devoted to space exploration of comets. The PROGRA2 experiment was funded by CNES; the parabolic flights campaigns were funded by CNES and the European Space Agency. S. Boresztajn and F. Piller (LISE/CNRS/Sorbonne University) are gratefully acknowledged for their SEM images.

\section{References}

Bardyn, A., Baklouti, D., Cottin, H., et al. 2017, MNRAS, 469, S712 Bentley, M. S., Schmied, R., Mannel, T., et al. 2016, Nature, 537, 73

Bertini, I., La Forgia, F., Tubiana, C., et al. 2017, MNRAS, 469, S404

Burchell, M. J., Fairey, S. A., Wozniakiewicz, P., et al. 2008, Meteorit. Planet. Sci., 43, 23

Carrillo-Sánchez, J. D., Nesvornỳ, D., Pokornỳ, P., Janches, D., \& Plane, J. M. C. 2016, Geophys. Res. Lett., 43, 11979

Dollfus, A., Bastien, P., Le Borgne, J.-F., Levasseur-Regourd, A.-C., \& Mukai, T. 1988, A\&A, 206, 348

Fink, U., \& Doose, L. 2018, Icarus, 309, 265

Francis, M., Renard, J.-B., Hadamcik, E., et al. 2011, J. Quant. Spectr. Rad. Transf., 112, 1766

Fray, N., Bardyn, A., Cottin, H., et al. 2016, Nature, 538, 72

Fray, N., Bardyn, A., Cottin, H., et al. 2017, MNRAS , 469, S506

Fulle, M., \& Blum, J. 2017, MNRAS, 469, S39

Fulle, M., Levasseur-Regourd, A. C., McBride, N., \& Hadamcik, E. 2000, AJ, 119, 1968

Fulle, M., Bertini, I., Della Corte, V., et al. 2018, MNRAS, 476, 2835
Glassmeier, K.-H., Boehnhardt, H., Koschny, D., Kührt, E., \& Richter, I. 2007, Space Sci. Rev., 128, 1

Goesmann, F., Rosenbauer, H., Bredehöft, J. H., et al. 2015, Science, 349, aab0689

Hadamcik, E., Renard, J. B., Worms, J. C., Levasseur-Regourd, A.-C., \& Masson, M. 2002, Icarus, 155, 497

Hadamcik, E., Renard, J.-B., Lasue, J., et al. 2007, J. Quant. Spectr. Rad. Transf., 106,74

Hadamcik, E., Levasseur-Regourd, A. C., Renard, J.-B., Lasue, J., \& Sen, A. K. 2011, J. Quant. Spectr. Rad. Transf., 112, 1881

Hadamcik, E., Lasue, J., Levasseur-Regourd, A. C., \& Renard, J.-B. 2019, Planet. Space Sci., in press

Hilchenbach, M., Kissel, J., Langevin, Y., et al. 2016, ApJ, 816, L32

Hörz, F., Bastien, R., Borg, J., et al. 2006, Science, 314, 1716

Kiselev, N., Rosenbush, V., Levasseur-Regourd, A.-C., \& Kolokolova, L. 2015, in Polarimetry of Stars and Planetary Systems, eds. L. O. Kolokolova, J. Hough, \& A. C. Levasseur-Regourd (Cambridge: Cambridge University Press), 379

Langevin, Y., Hilchenbach, M., Ligier, N., et al. 2016, Icarus, 271, 76

Lasue, J., Levasseur-Regourd, A.-C., Fray, N., \& Cottin, H. 2007, A\&A, 473, 641

Lasue, J., Levasseur-Regourd, A. C., Hadamcik, E., \& Alcouffe, G. 2009, Icarus, 199, 129

Lasue, J., Levasseur-Regourd, A.-C., \& Lazarian, A. 2015, in Polarimetry of Stars and Planetary Systems, eds. L. Kolokolova, J. Hough, \& A. C. Levasseur-Regourd (Cambridge: Cambridge University Press), 419

Levasseur-Regourd, A. C., Mann, I., Dumont, R., \& Hanner, M. S. 2001, in Interplanetary Dust, eds. E. Grün, B. A. Gustafson, S. Dermott, \& H. Fechtig (Berlin: Springer), 57

Levasseur-Regourd, A.-C., Zolensky, M., \& Lasue, J. 2008, Planet. Space Sci., 56,1719

Levasseur-Regourd, A.-C., Renard, J.-B., Shkuratov, Y., \& Hadamcik, E. 2015, in Polarimetry of Stars and Planetary Systems, eds. L. Kolokolova, J. Hough, \& A. C. Levasseur-Regourd (Cambridge: Cambridge University Press), 62

Levasseur-Regourd, A.-C., Agarwal, J., Cottin, H., et al. 2018, Space Sci. Rev., 214,64

Mannel, T., Bentley, M. S., Schmied, R., et al. 2016, MNRAS, 462, stw2898

Markkanen, J., Agarwal, J., Väisänen, T., Penttilä, A., \& Muinonen, K. 2018, ApJ, 868, L16

Matrajt, G., Ito, M., Wirick, S., et al. 2008, Meteorit. Planet. Sci., 43, 315

Moreno, F., Guirado, D., Muñoz, O., et al. 2018, ApJ, 156, 237

Muñoz, O., \& Hovenier, J. W. 2015, in Polarimetry of Stars and Planetary Systems, eds. L. Kolokolova, J. Hough, \& A. C. Levasseur-Regourd (Cambridge: Cambridge University Press), 130

Muñoz, O., Volten, H., De Haan, J. F., Vassen, W., \& Hovenier, J. W. 2000, A\&A, 360, 777

Nesvornỳ, D., Jenniskens, P., Levison, H. F., et al. 2010, ApJ, 713, 816

Renard, J.-B., Worms, J.-C., Lemaire, T., Hadamcik, E., \& Huret, N. 2002, Appl. Opt., 41, 609

Rowan-Robinson, M., \& May, B. 2013, MNRAS, 429, 2894

Tomeoka, K., \& Buseck, P. R. 1988, Geochim. Cosmochim. Acta, 52, 1627

Worms, J.-C., Renard, J.-B., Hadamcik, E., Levasseur-Regourd, A.-C., \& Gayet, J.-F. 1999, Icarus, 142, 281

Worms, J.-C., Renard, J.-B., Hadamcik, E., Brun-Huret, N., \& LevasseurRegourd, A. C. 2000, Planet. Space Sci., 48, 493 